\title{
El redescubrimiento por la psiquiatría de los disminuidos psíquicos ${ }^{1}$
}

\begin{abstract}
RESUMEN: Un porcentaje significativo de los disminuidos con trastorno psiquiátrico en casa o en servicios sociales estan desprovistos de cuidados adecuados.

PABLABRAS CLAVE: disminuidos con trastornos psiquiátricos; normalización; tratamiento especializado; Paises Bajos; España.
\end{abstract}

\begin{abstract}
SUMMARY: A significant percentage of mentally retarded with psychiatric disorders, at home or in social services, are in want of appropriate care.

KEYWORDS: mentally retarded with psychiatric disorders; normalization; special treatment; the Netherlands; Spain.
\end{abstract}

\section{Introducción:}

Hasta los años 60 del siglo pasado, en la mayoria de los paises, los disminuidos psiquícos (personas con discapacidad, retraso mental) vivían en los hospitales psiquiátricos entre otros pacientes psiquiátricos. Aunque ya existían instituciones particulares para disminuidos, incluso escuelas especiales, todavía muchas personas con oligofrenia eran considerados enfermos psiquiátricos. En los ultimos 40 años la población de los manicomios ha cambiado mucho, tanto por la deshospitalización general, como por la aplicación de una política selectiva que limita los ingresos en los hospitales. Los viejos, los psicópatas, los alcohólicos y también los oligofrénicos han dejado de pertenecer a la clientela asilar, engrosando las filas de nuevas instituciones, dependientes ahora de los servicios sociales (1). Datos procedentes de los registros de casos nos muestran la salida de los hospitales psiquiátricos de los disminuidos en los años setenta en Holanda, y en otros paises (2). Con esto los disminuidos desaparecieron del ámbito de la psiquiatría aunque hubieron psiquiatras, muy pocos, que los siguieron en servicios particulares. El conocimiento de los trastornos psiquiátricos de los disminuidos disminuyó, La oligofrenia quedó, sin embargo, en la clasificación diagnostica de psiquiatria (3). Además, muchos disminuidos estan igualados asimismo con otros trastornos psiquiátricos, muchas veces más que otras personas (4). Un porcentaje significativo de los disminuidos necesita tratamiento psiquiátrico que no tiene en este momento. De esto trata este artículo; de los problemas de conducta de los disminuídos y detrás de qué trastornos psiquiátricos pasan desapercibidos a veces.

Empezaré -en lo que pueda conocer- dibujando a grandes rasgos la situación actual de los disminuidos en Holanda y en España. A continuación entraré en detalles historicos contemporáneos y en el desarollo de los "equipos de consulta" que ha tenido lugar en Holanda en los años noventa, seguido de una discusión acerca de la relación entre los servicios de salud mental y los servicios sociales en ambos paises. Concluiré haciendo ver la necesidad del redescubrimiento por parte de la psiquiatría de los disminuidos. 


\section{La situación actual}

En Holanda hay 101.800 disminuidos (5) y en España 160.614 (6). Estas cifras proceden de los contactos que los pacientes establecen con los servicios. El porcentaje de disminuidos respecto a la población general es mayor en Holanda que en España: 6,53 por cada mil en comparación con un 4,07 \%o $(7,6)$ (Tabla 1). Quizás, sea mayor el porcentaje en Holanda porque en esta país es menos tabú y un servicio ambulatorio como el "Servicio Pedagógico Social" (SPD) no existe en España. La mayor parte de los disminuidos en España viven en casa: $97 \%$ en comparación con $49 \%$ en Holanda. Por otro parte hay más disminuidos cuidados en los hospitales psiquiátricos en España (cerca de 3000) que en Holanda (cerca de 1000). Los disminuidos constituyen actualmente un grupo pequeño del total de los pacientes ingresados en hospitales psiquiátricos en Holanda: 1000 en 23.000 camas, de las que la mitad están ocupadas por pacientes crónicos $(8,9)$.

\section{Historia contemporánea}

Aunque los pedagogos y psicólogos trataron de introducir un nuevo modelo, llamado el modelo de desarrollo o cultura, desde la mitad de los años sesenta, en los servicios de disminuidos en Holanda el modelo médico siempre fué dominante hasta 1975. Carel Muller, asimismo psicólogo, criticó los dos modelos poniendo el acento en la limitacion del individuo. Ségun Muller, se debería tratar los disminuidos como personas sin discapacidades, y ofrecerles un lugar normal en la sociedad. Aunque Muller no ha conseguido convencer a la administración de sus ideas, el "asunto de Dennendal" significó un cambio en la forma de pensar tradicional sobre los "psicasténicos o debiles". Los principios de "normalización" y "derechos iguales" (tratarle a uno de igual a igual), ya más común en los paises escandinavos a la sazón, marcaron el desarrollo de los cuidados para los disminuidos en Holanda a partir de 1975.

Una fotografia - en los periódicos y en la televisión- estremeció (despertó) al país quince años después. Era una fotografia de una mujer desnuda encadenada al muro en un servicio social. Vergonzosa y asustada, la gente se preguntó como pudo ser posible en una sociedad tan próspera y progresista como la holandesa. Una investigación mostró que habían aproximadamente 3000 disminuidos en los instituciones residenciales con problemas de conducta muy graves: muchos permanecieron en iguales condiciones, aislados o atados la mayor parte del dia. A un número importante de ellos les faltaba un diagnóstico claro y no eran tratados convenientemente. Una gran desventaja para la "normalización" de estos disminuidos muy graves y con doble diagnóstico se puso de manifiesto. Faltan programas adecuados y medios para varias razones (10).

\section{Los equipos de consulta}

La respuesta política a esta situación, desde 1990, fue crear equipos de consulta para disminuidos con problemas graves. Son equipos regionales, múltidisciplinarios y tienen la mayoría de las veces también un psiquiatra. La exacta composición del equipo depende cada vez más de los problemas del disminuido en cuestión. Un equipo como éste 
aconseja al personal de las instituciones residenciales y desarrolla un plan de tratamiento individual para personas con retraso mental y con grandes problemas de conducta. El equipo tiene también medios en caso de necesidad para la realización del plan.

Los equipos han visto desde entonces a cientos de estas personas. Casi la mitad de ellos se provoca autolesiones, muchas veces con daños fisicos irreversibles. Muchas de estas personas tienen trastornos psiquiátricos, como psicosis, esquizofrénia, depresión o 'autismo'. La experiencia de estos equipos, y también de secciónes especiales que han sido fundadas en colaboración entre los servicios sociales y los servicios de salud mental, subraya la necesidad de servicios de tratamiento especializado para disminuidos con doble diagnóstico. Necesitan profesionales con conocimiento y experiencia con ellos, sobre todo psiquiatras. Además, estos profesionales deben trabajar no sólo para personas con doble diagnóstico en servicios residenciales, sino también para aquellos que viven en sus casas.

También en España hay un nuevo concepto de retraso mental (11-14). Y dicen también que hay una mala coordinación entre los servicios sociales y de salud mental, que deja lagunas en el cuidado de estas personas con retraso mental (15), (16). Una investigación española (17) muestra que 33\% de las personas con retraso mental tiene también un trastorno psiquiátrico. Que yo sepa, hay pocos servicios especializados: solamente algunos en Cataluña, Andalucia y Galicia (18). El INSERSO (12) financia desde hace poco tratamientos con psicoterapia, pero por regla general falta bastante atención para esto grupo con doble diagnóstico, especialmente por parte de la psiquiatría y psicología clínicas.

\section{Discusión}

He trabajado sobre todo en los registros de casos. Los últimos diez años he trabajado también en el sector de cuidados para las personas con retraso mental, en la administración y la investigación. A mi me parece bien que resurja este nuevo concepto de retraso mental. La integración en la sociedad para la mayoría de las personas con retraso mental, es posible. Quizás la psiquiatria puede aprender de eso.

Pero tengo también mis preocupaciones sobre la minoria de las personas con retraso mental. En parte se quedan todavía en los hospitales psiquiátricos, más en España qué en Holanda, pero, me pregunto ¿con un cuidado adecuado en todos los casos? Por otro parte, muchas personas con retraso mental y trastorno psiquiátrico que viven en sus casas o en servicios sociales están también desprovistos de cuidados adecuados. Hace falta un oído fino y ojos de psiquiatra, con bastante conocimiento de psicofarmacología entre otras cosas. No digo que lo mejor sea cuidarlos en los servicios de salud mental; necesitan la psiquiatría, pero quizá más en su medio natural. Al mismo tiempo le puede proporcionar al profesional interesado la posibilidad de descubrir nuevas cosas. Estoy segura de que los psiquiatras que se interesen por los disminuidos complicados tendrán un trabajo duro pero, a su vez muy satisfactorio. No hay más remedio, hay aquí trabajo para psiquiatras. 
TABLA 1:

NÚMERO DE DISMINUIDOS EN ESPAÑA Y EN HOLANDA POR EDAD: en \%o

\begin{tabular}{|lcccc|}
\hline Edad: & $<18$ & $18-64$ & $>64$ & total \\
\hline España* & 1,24 & 2,65 & 0,179 & 4,07 \\
\hline Holanda** $^{*}$ & 1,99 & 4,01 & 0,545 & 6,53 \\
\hline
\end{tabular}

$*(6)$

$* *(7)$

\section{BIBLIOGRAFIA}

1. Espinosa Iborra, J. "Repuesta social e institutional al problema del enfermo mental cronico: una revision historica" En: Julián Espinosa (coord.) Cronicidad en psiquiatria, Madrid, AEN p.15- 72.

2. Horn, G.H.M.M. ten y otros (eds.). Registros de casos psiquiatricos en salud publica: un inventario mundial 1960-1985., Santiago de Compostella, Xunta de Galicia, 1989.

3. DSM. Diagnostic and Statistical Manual of Mental Disorders, fourth edition, Washington, American Psychiatric Association (APA), 1994.

4. Dosen, A., Menolascino, F.J. (eds.). Depression in Mentally Retarded Children and Adults, Leiden, Logon Publications, 1990.

5. Centraal Bureau voor de statistiek. Vademecum gezondheidsstatistiek 1998, The HagueSdu publisher, CBS-publications, 1998.

6. INSERSO (Instituto Nacional de Servicios Sociales), Las personas con minusvalia en España: aspectos quantitativos. Madrid: Ministerio de Asuntos Sociales-INSERSO, 1989.

7. Klerk, M.M.Y. de \& Timmermans, J.M. (eds.), Rapportage gehandicapten 1997, Leefomstandigheden van mensen met lichamelijke of verstandelijke beperkingen, The Hague, Sociaal en Cultureel Planbureau, 1997.

8. Schnabel, P. "The mental health services: more than Psychiatry alone", In: A.J.P.Schrijvers (ed.), Health and Health Care in the Netherlands, Maarsen, Elsevier/De Tijdstroom, 1997, p.119-131.

9. Horn, G.H.M.M. ten. "Care for people with a Mental Handicap", In: A.J.P. Schrijvers (ed.), Health and Health Care in the Netherlands, Maarsen, Elsevier/De Tijdstroom, p. 132-140.

10. Klapwijk, E.Th. "The Netherlands: description of general services for people with a mental handicap", Journal of Intelectual Disability Research, 1993, 37, supplement 1, p.44-46.

11. FEAPS, Manual de actividades de apoyo a las familias de personas con deficiencia mental \& FEAPS, Un nuevo concepto de Retraso Mental

12. IMSERSO (Instituto de Migraciones y Servicios Sociales), Plan de Acción para Personas con Discapacidad 1997-2002. Madrid: Ministerio de Trabajo y Asuntos Sociales, 1997 


\section{INFORMES}

13 Lacaste Reoyo, J.J. and FEAPS, “Apoyo a las familias de las personas con minusvalias”. In: $L a$ discapacidad en el siglo XXI, Plan de acción. Colección Rehabilitación de INSERSO.

14. Pérez Martin, J., Alonso Trujillo, F., Salvador Carulla, L. Integración y Promoción de las personas con Retraso Mental. Cordoba: PROMI (internet), 1997.

15. Salvador Carulla, L. "Mental Health Services in Spain”, In: M. Moscarelli, A. Rupp and N. Sartorius. Handbook of Mental Health Economics and Health Policy, volume I, Schizophrenia, John Wiley and sons Ltd., 1996.

16. Salvador Carulla, L. and Martínez-Maroto, A. "Spain: description of general services for people with mental handicap in Spain". Journal of Intellectual Disability Research, 1996, 37, supplement 1, 34-37.

17. Garcia-Mellado, M.J. y otros. "A reliability study of the Spanish version of the Social Behaviour Schedule (SBS) in a population of adults with learning disabilities", Journal of Intellectional Disability Research, 1998, 42, part 2: 22-28.

18. Verdugo Alonso, M.A. \& Jordán de Urríes Vega, F.J. (eds.) Hacia una nueva concepción de la discapacidad. Salamanca: Amarú ed.. 1999. Con: 1.Díez Alvarez, A. Estudio de centros: una aproximación de las personas con discapacidad en Castilla y León. 2. García Ibañez, J., Santacana, I. \& Ramo, R. La atención en salud mental a las personas con restraso mental en Cataluña. 3. Rodríquez-Blázquez, C. Salvador Carulla, 1. Romero, C. \& Atienza, C. Necesidades de atención a personas con retraso mental y trastornos psiquiátricos en España. El informe Biomed/Aldapt 1998.

\section{*Sociologa}

Correspondencia: Dr. G.H.M.M.(Sineke) ten Horn

Nico Rostlaan 5

9721 ZN Groningen

Tel: +31 505257917

Email: sineke.tenhorn@planet.nl

Fecha de recepción: 17-XII-01 